\title{
Childhood Maltreatment and Exposure to Domestic Violence at Infancy and Adolescence: What Is Left Into Adulthood?
}

\author{
Tânia B Cavaco ${ }^{1}$, Raquel Campos ${ }^{1}$ \\ ${ }^{1}$ Child and Adolescent Psychiatry Resident, Garcia de Orta Hospital (Almada, Portugal) \\ Institutional e-mail: tania.cavaco@hgo.min-saude.pt
}

\section{INTRODUCTION}

* About $\mathbf{2 5 \%}$ of adults worldwide have been exposed to some form of domestic violence (DV) in infancy;

- Childhood maltreatment and exposure to DV may include physical or psychological abuse, parent-child neglect, or witnessing intimate partner violence, which holds a long-term adverse impact on a social, emotional, behavioural, psychological and physical level.

* These traumatic events are linked to multiple deleterious outcomes, development of psychopathology and perpetration of violence;

* The severity of these consequences from childhood into adulthood, and their association to transgenerational cycles of violence, set the importance of a professional, optimized prevention and intervention.

\section{METHODS}

Non-systematic review of original and revision articles, indexed on Pubmed, in English and Portuguese, selected by relevance according to the keywords: domestic violence, childhood, maltreatment, psychopathology, child abuse, neglect. Search was extended to articles referenced on the bibliography.

\section{RESULTS}

* Childhood maltreatment and DV are a strong predictor of perpetration of violence (risk $\uparrow \mathbf{3 x}$ ) and lower life satisfaction, in adulthood;

* The duration of violence has a greater impact on children's distress and wellbeing, than its severity;

* Parents who are victims of DV may not be physically nor psychologically capable of offering a safe, stable and peaceful environment;

* Risk Factors for maltreatment and DV include low socioeconomic status, black ethnicity and genetics (explains $44-47 \%$ of variance);

* Repeated exposure to violence might lead to emotional desensitisation.
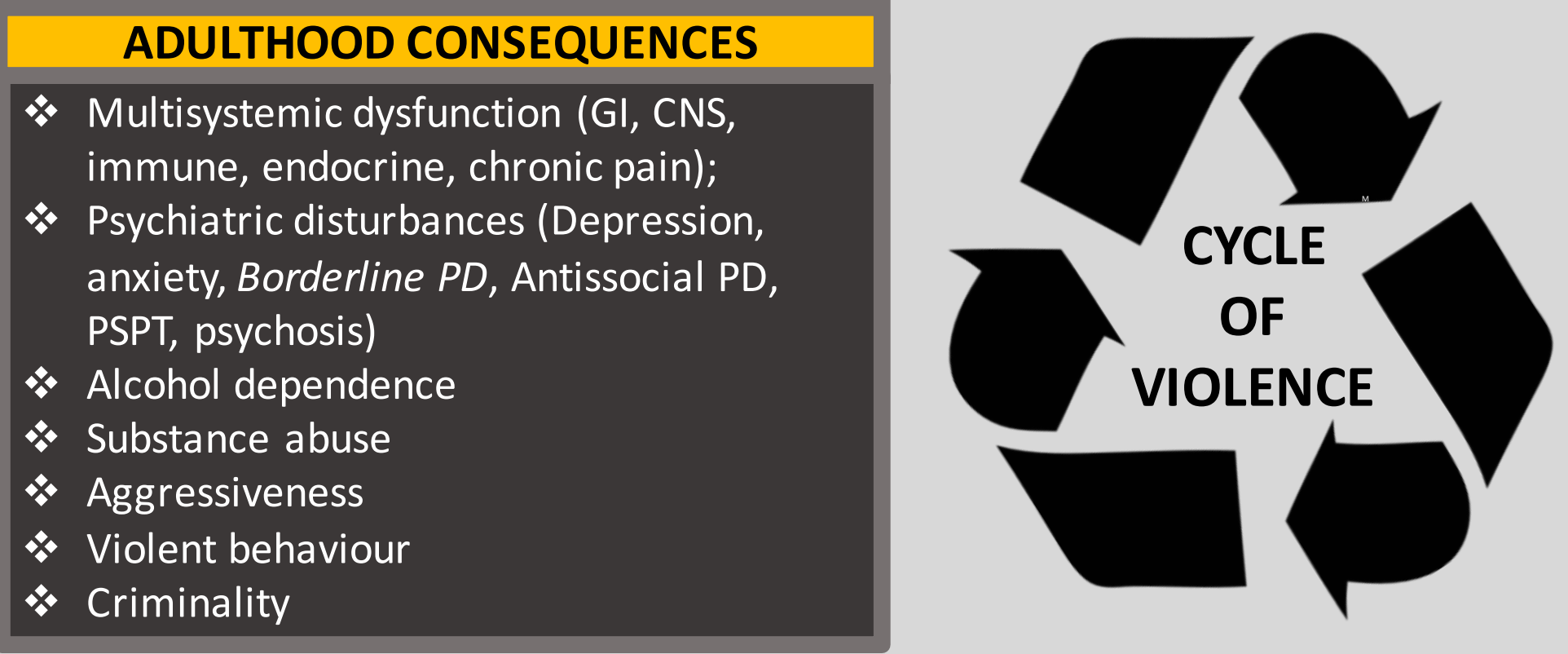

CHILDHOOD AND ADOLESCENCE CONSEQUENCES

* Behavioural (internal- or externalizing symptoms)

* Social (dysfunctional interpersonal relationships, defensive avoidance, isolation)

* Cognitive (development and learning deficits)

* Emotional (insecure attachment, affective dysregulation, regression, undervalued or fractured self, aggressiveness)

* Psychiatric (separation anxiety, generalized anxiety, depression, disruptive conduct, self-harm)

Physical (traumatic lesions, organic disorders)

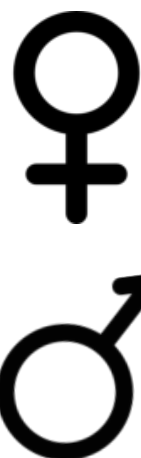

GIRLS: ++ internalise symptoms (withdrawal, anxiety, depression)

BOYS: ++ externalise symptoms, violence against peers, antisocial behaviour
CHILDREN 1-4 Y.O.: Sleep problems, separation anxiety, repetitive play, regressive behaviour, impaired understanding, tantrums, tune out noise, inhibited independence.
CHILDREN 5-10 Y.O.: Separation anxiety, factitious disorder dependence, withdrawal, sleep disorders, attention deficit, depression, insecurity, schoo problems, bullying, guilt, aggressiveness, hypervigilance.
CHILDREN/ ADOLESCENTS 11-16 Y.O.: Self-blame, eating disorders, depression, suicidal ideation, substance abuse, self-harm, criminality, poor social networks, disaffection with education.

\section{CONCLUSION}

* Witnessing DV and childhood maltreatment have deleterious and longstanding effects on mental and physical health; however, stereotyping children as permanently and inevitably damaged is erroneous;

* The development of preventive strategies, early intervention and protective measures against the perpetration of violence is essential;

* PRIMARY PREVENTIVE STRATEGIES: Prevention of witnessing DV and DV per se; Identification of families at higher risk and the establishment of preventive measures to support new parents;

* PREVENTION STRATEgIES: (1) Psychosocial intervention might include improved self-care, coping strategies, self-harm reduction, bonding strategies (parents); Children's shelters; (2) Psychotherapeutic intervention might revert epigenetic modulations caused by chronic stress and child victimization; Parental psychotherapy and support; CBT for children. 